\title{
Supporting the Use of Social Media by Emergency Managers: Software Tools to Overcome Information Overload
}

\author{
Runqing Rao \\ New Jersey Institute of \\ Technology \\ rr434@njit.edu
}

\author{
Linda Plotnick \\ Jacksonville State University \\ lplotnick@jsu.edu
}

\author{
S. Roxanne Hiltz \\ Centre for Integrated Emergency \\ Management, University of \\ Agder, Norway \\ roxanne.hiltz@gmail.com
}

\begin{abstract}
Information overload has been suggested as a potential barrier to use of social media by emergency managers, especially for gathering information during disasters. This paper, based on a survey of 477 U.S. county-level emergency managers, examines the relationship of the perception of information overload as a barrier to social media use for gathering information, to the intention to use social media. It also examines the relationship of perceived usefulness of certain technological advancements currently being developed to intention to use. Results indicate that emergency managers' perception of information overload as a barrier to use is negatively related to intention to use it, while perceptions of the usefulness of these technologies are positively related to intention to use. The study calls attention to the importance of resolving information overload to increase the use of social media for emergency management, and suggests the implementation of appropriate software tools to ameliorate this problem.
\end{abstract}

\section{Introduction}

A combination of climate change and social behavior has led to increasing threats of damages from natural and man-made disasters ${ }^{1}$. For instance, the frequency of weather-related catastrophes such as windstorms and floods has had an increasing influence on disaster trends in recent decades, particularly putting global coastal populations at risk as sea level continues to rise ${ }^{2}$. In addition, jihadists have adopted a strategy of trying to inflict massive citizen casualties in

\footnotetext{
${ }^{1}$ In this paper, we will refer somewhat interchangeably to major emergencies, disasters, catastrophes, and crises; the distinction is the scale of the disruption, but social media can be used for events and incidents that range from the local to the multi-national. We mean to encompass all events that are too large or serious to be handled by just the local first responders.

${ }^{2}$ http://www.worldwatch.org/node/4127
}

their attacks around the world. Thus, to help create a safer and more secure world, it is important to understand how information systems may best be used to improve the preparation for and response to crises by both the public and professional emergency managers (EMs).

Increasingly, the public uses social media (SM) to record and share information about unfolding disaster events, with text, pictures and video. Studies have demonstrated that the information in them can be valuable for improving situational awareness by providing timely, actionable information about an evolving disaster [8, 14]. However, potential information overload due to the flood of hundreds of thousands or even millions of posts during large scale emergencies is an important problem [17].

During the last decade, research has burgeoned on software solutions to enable the identification, classification, organization, and assessment of SM posts that could aid emergency managers. There are scores of prototype systems, often developed as academic research with little or no consultation or participation with EMs to determine if the innovation would be attractive to them. Few of these prototypes have been developed to an operational level where they are actually deployed and used in real-time during major crises, and none have become "standard" for U.S. government organizations. These tools differ in their purposes and approaches but many of them are useful to create categories of related data. The grouping together of related data, called "chunking" by Miller [16] is the overarching approach to improving SM effectiveness and use this study addresses.

This paper is based on survey responses from 477 county-level EMs in the U.S. It examines their perceptions of the extent to which information overload is a barrier to their use of social media (SM) for collecting information from the public during crises and their reactions to several potential software innovations that exist in prototypes and might help overcome some of those barriers. The overarching goal 
of the series of studies of which this paper is a part is to identify the most important barriers and potential mitigations of these barriers, so that technologists, policy makers, and the Public Information Officers or others in charge of communication related to emergencies will be able to address the issues together and make it possible to fully exploit the potential of SM during disasters, both for dissemination of information and for obtaining information from the public about conditions and needs.

The first study in this series consisted of semistructured interviews with emergency managers (EMs) to identify barriers that may be preventing them from full use of SM in their agencies and to discuss what might help mitigate these barriers [25]. The results of that study informed our development of an online survey to address EMs' concerns about barriers to both collecting and disseminating information via SM, policy issues, and agency goals when SM is used [17]. The results of the analysis of the study, reported in [17], suggest that the greatest hesitation to use SM is for the purpose of collecting public SM data. We then developed a survey that focused on these barriers to collect public SM data and potential solutions to the most grievous barriers. This paper focuses on an analysis of the responses to this most recent survey.

Our research questions are:

RQ1: To what extent is information overload perceived by emergency managers as an important barrier (BIO) to the use of SM to gather information during a disaster?

RQ2: What is the relationship between $\mathrm{BIO}$, and behavioral intention to use SM (IU) in the future?

RQ3: To what extent is the perceived usefulness of several software tools (Chunking) by emergency managers related to their intention to use social media in emergency management (IU)?

RQ4: To what extent is Behavioral Intention to Use SM for gathering information by emergency managers (IU), predicted by a model that includes the extent to which information overload is perceived as an important barrier (BIO); and the perceived usefulness of "chunking" software tools?

In the remainder of this paper, in section 2 we review the concept of information overload as it applies to the use of SM and then briefly describe some of the most promising technical "solutions" to this problem that have appeared in the literature. The methods and findings for this study are described and discussed in Sections 3 and 4. We conclude with limitations, and future research recommendations.

\section{Literature Review and Hypotheses}

\subsection{The Information Overload Issue}

Information overload or the sheer volume of the data stream, which exceeds human processing capacity, has been identified in past research as one of the major barriers to the use of social media for gathering information during a disaster [6]. It has traditionally been defined as "information presented at a rate too fast for a person to process" [18]. Besides "too fast," a second dimension of information overload is "too much," given the limitations of human shortterm memory. In a classic paper, Miller [16] showed that for a number of human cognitive and perceptual tasks, channel capacity is between 5 and 9 items.

Miller's "magical number seven plus or minus two" has been noted by Eppler and Mengis [4] as a very significant perspective when considering information overload. Miller describes several techniques for trying to mitigate this limitation, including the idea of "organizing or grouping the input into familiar units or chunks" [16 p. 93]. The idea is that more information can be processed, remembered, and understood, if it is "chunked" so that the individual pieces of information in a chunk are related. Miller refers to the amount of information as "variance" and "amount of transmitted information" (received) as "covariance or correlation" [16]. Miller claims that the critical dimension of the information is the number of chunks and not the amount of data contained within each chunk. It is noteworthy that in his article, Miller discusses an experiment that showed that when subjects were told ahead of time what attributes were being considered with data, they made more accurate judgements on the attributes than when they were informed of which attributes were to be judged after having observed the data [16]. This suggests that technological enhancements to SM that "chunk" social media data into groupings that are identifiable by the user prior to actual examination of the data may be useful to combat information overload. The technology would be doing what in his 1956 seminal paper Miller called "recoding" which he claimed "is an extremely powerful weapon for increasing the amount of information that we can deal with." [16 p. 95]

That "chunking" is an effective way to mitigate the natural human limitations on the amount of data that can be retained in short term memory, processed, etc. has been suggested by other researchers as well. For example, Tegarden [20] reports that visualizations can help in chunking information in a way that increases the amount of information people can retain. Koroleva, Krasnova, and Günther [12] found that users want this 
chunking to be done automatically - i.e. a call to technological approaches to chunking.

Eppler and Mengis [3] note that Miller's work on the limitations of human information processing capacity has been the foundational theory of empirical research in fields such as marketing, accounting, etc. In their later comprehensive review of the information overload literature, Eppler and Mengis [4] found that technological tools are often mentioned in the literature as countermeasures to the problem of information overload. Some of the measures touted in the articles reviewed by Eppler and Mengis [4], in fact, do some form of chunking (e.g. aggregation, categorization, visualization, organization, filing).

An early exploration of the information overload problem in Computer-Mediated Communication [7 p. 682], describes the issue in a way that still applies to the use of SM in crisis management:

$\ldots$ in the context of CMC... the term refers first

to the delivery of too many communications and to an increase in social density that gives individuals access to more communications than they can easily respond to, and second to what might be termed information entropy, whereby incoming messages are not sufficiently organized by topic or content to be easily recognized as important or as part of the history of communication on a given topic.

In other words, a third dimension of information overload is the extent to which the information is organized and categorized. Users can deal with more information if it is "chunked" and organized in some way by the system.

Even under non-crisis conditions, users of social media often experience information overload. For example, a study of Facebook users [2] found that the average user sometimes feels overloaded by the "news feed" structure. As Verma, et al. [21 p. 385] state, the problem for emergency managers:

So much information is now broadcast during mass emergencies that it is infeasible for humans to effectively find it, much less organize, make sense of, and act on it. To locate useful information, computational methods must be developed and implemented to augment human efforts at information comprehension and integration.

This paper explores the extent to which perceived usefulness of categorization ("chunking") tools for incoming SM posts is related both to perceptions of the extent to which information overload is a barrier to use of social media by emergency managers, and to their intention to use social media on the job, in the future.

\subsection{Software Innovations: Major Streams of Research}

There are now scores of studies in the literature that describe possible software enhancements and systems that could improve the usability and usefulness of social media for disaster management. See Imran et al. [8] for a complete survey of these technologies. We review a few of the major streams of research on this topic, which served as a basis for our listing and describing potential software solutions to U.S. public sector EMs in our studies. We note that human help, in the form of volunteer groups (VOSTS) has been successfully used in disasters, such as the Colorado floods of 2013, to help gather and organize SM information. [24]. However, this study focuses on technology assistance research as we believe that, once available, technological solutions will be more available and able to perform more complex solutions than volunteers alone.

\subsubsection{Managing the Flood of Data: "Natural} Language Processing to the Rescue?". A necessary step in making sense of SM data is to organize or classify the information into useful categories. SM are to some extent self-organizing. For example, Twitter users invent and use hashtags to describe topics, and Facebook users establish and name new groups to discuss topics. However, the basic organizing tools and conventions for public SM use are inadequate for the purpose of sensemaking by EMs.

The problem in using NLP (Natural Language Processing) is that user-generated content is intrinsically noisy and embodies language uses that are markedly different from conventional documents (e.g. news articles, blogs), which makes NLP techniques less applicable [23]. Verma et al. [21] describe a program developed to automatically identify messages communicated via Twitter that can contribute to situational awareness, which is operationally defined as messages that contain tactical, actionable information based on specific details about the scope, nature, and location of problem situations. The system used a combination of hand- annotated and automaticallyextracted linguistic features. Another example is work by Cameron, Power, Robinson, and Yin [2] (see also [23]) that describes work with the Australian Government Crisis Coordination Center to detect, assess, summarize, and report messages of interest for crisis coordination published by Twitter. They developed a platform and client tools, collectively termed the Emergency Situation Awareness (ESA) system, to demonstrate how relevant Twitter messages can be identified and utilized to inform the situation 
awareness of an emergency incident as it unfolds. $\left(\mathrm{see}^{3}\right)$. The system automatically classifies tweets as "interesting" or not, based on whether they identify infrastructure damage; then it uses a clustering engine to gather and visually display sets of tweets related to an incident.

Related work and systems for automatic classification and extraction of useful information from tweets has been conducted by a group of researchers at the Qatar Computing Research Institute [9,10,11]. Their objective is to build and test a system that will automatically extract and classify valuable (useful) "information nuggets" (brief, self-contained information items relevant to disaster response) from microblog posts, to provide actionable information that will accelerate disaster response. They refer to their system as a "Twitter dashboard for disaster response". The purpose of this experimental dashboard is to provide humanitarian organizations with a platform that will enable them to create their own automatic classifiers on the fly.

2.2.2. Visualization and Geo-Tagging Features for Sensemaking. Even if a system identifies most relevant SM posts and rates their probable trustworthiness and classifies them into groupings, long lists of such items would still not be very comprehensible to human managers. The data need to be presented in the form of mapping and visualizations if they are truly to contribute to sensemaking.

Mapping of crowd sourced information in disaster response gained a lot of media attention as a useful tool during the deployment of the Ushahidi Crisis Map system in Haiti in 2010 [19]. MacEachern et al. [15] describe a prototype system that incorporates "geoanalytics"; a system that includes analysis and visual displays from analyzed Twitter posts in the form of a map, to aid in situational analysis for crisis management. This work [15] also included a survey of emergency managers which addressed not only what SM they currently use, but also the tools and features they would like to have to more effectively incorporate SM into their situational awareness and decision making activities. Among the most popular choices for features to include were Maps (95\%), photos/video collections (71\%), time graphs $(60.5 \%)$, keyword clouds $(58 \%)$ and clustering tools (47\%).

The ESA (Emergency Situation Awareness) system described above also includes a geo-tagging component that displays the content of a tweet at its geographic location on a map. If a tweet is already geotagged by its author, its latitude/ longitude coordinates

\footnotetext{
${ }^{3}$ https://esa.csiro.au/ausnz/index.html
}

are used directly; otherwise, the location field of the user profile is used to determine a latitude/longitude position [22].

\subsection{Hypotheses}

Based on the literature review, our hypotheses are:

H1: The extent to which information overload is perceived as a barrier to use of Social Media for gathering information during emergency management $\mathrm{BIO}$ ), will predict, in a negative direction, the behavioral intent of emergency managers to use social media on the job in the future (IU).

H2: The level of the perceived usefulness of "chunking" tools for incoming social media (Chunking), will predict, in a positive direction, the behavioral intent of emergency managers to use social media on the job in the future (IU).

H3: The extent to which information overload is perceived as a barrier to use SM (BIO) will predict the perception of usefulness of the chunking technologies proposed.

\section{Methodology for this Study}

We developed a survey based upon the research questions, literature review, and results of analysis of the prior interviews and survey conducted by the researchers and summarized above [25, 17].

\subsection{Survey Development and Measures}

This survey focuses on both the barriers to SM adoption for collecting data by government agencies and the agency managers' perceptions of the usefulness of technological enhancements currently being developed. The survey was approved by a University IRB, and distributed using the online survey application SurveyMonkey®.

We repeated questions from a prior survey about barriers to collecting data, in order to be able to correlate these concerns with attitudes towards potential software tools to deal with some of them, and also because we anticipated that the response rate would be better for this second, shorter survey. For each proposed barrier and enhancement, there was a single 7-point semantic differential scale item (question) with an additional possible answer of "Don't Know". This was to ensure that all questions were read by the respondents. However, the "Don't Know" answers were excluded from analyses other than measures of frequency of responses. We needed to keep this survey brief because EMs are very busy people and will tend not to answer a survey that looks 
long, or to stop answering in the middle of a survey that takes more than about ten minutes. Skip logic available in SurveyMonkey®, which allows for semicustomization of survey question presentation based on individual responses, was used to assure that respondents were only presented with relevant questions. The wording for the items used to measure the constructs in this study are presented below in the order in which they appeared in the survey. The item that measured the extent to which information overload is perceived as a barrier (BIO) is:

Please indicate the extent to which each of the following are barriers to social media use for collecting information for your agency...

Barrier of Information Overload (BIO): (1-7 check boxes for not a barrier to a large barrier, plus Don't Know)

The items for rating the usefulness of potential software applications are shown in the factor analysis table in the Results section; The response scale ranged from "Not useful" (1) to "Very useful" (7):

The question for Behavioral Intention to Use (IU) is:

Assuming that I have, or could have, access to social media and permission to use it in my job, I Definitely do) intend to use it. (1, Definitely do not 7,

In addition, there were questions pertaining to agency and respondent characteristics and a number of response boxes for comments.

\subsection{Participants}

We used the U.S. Census Bureau web site (www.census.gov) to develop a list of the 3000+ county level Emergency Management Agencies (EMAs) in the United States. Counties were used as the unit of analysis because they are primary implementers of emergency management programs in the U.S. and when Federal declarations are made for disaster assistance it is at the county level.

Invitations were sent out to the 2,858 EMA directors or coordinators for whom email addresses were found, with a link to the survey, between April and September of 2014. There were 530 responses of which 477 were usable. The remaining 53 responses were deleted because the respondents did not answer any questions regarding barriers or enhancements. It should be noted that not all responses kept in the data set for analysis had responses to all questions. Therefore, in the analysis the sample size may be less than 477 for a particular test.

Overall, the response rate was $8 \%$ of all counties, but was higher for the counties larger in terms of size of the population served $(23 \%$ of large counties emailed). The particularly low (6\%) response rate for the agencies serving the counties with the smallest population is understandable because many of these managers also have other full time positions such as sheriff or firefighter; they have very little time to spend on their EM work. Despite the low response rate, there were over 100 responses for this category, so they are well represented.

\subsection{Data Analysis}

Data analysis was carried out using the SPSS 22.0 statistical package [13]. To determine which technology enhancement items were indicators for the same construct, we performed factor analysis with Varimax rotation of all 11 technological enhancements proposed in the survey. Two factors emerged. The factor of interest to this research was the one identified by the researchers as advancements that provide "chunking" of data as described by Miller [16]. For this factor, Cronbach's alpha test to assess the convergent validity and internal consistency of the items in the "Chunking" scale was performed. Descriptive statistics were used to visualize variable distributions; and correlation, and multiple regression were conducted to examine the hypotheses.

\section{Results}

In this section, we first describe the characteristics of the survey respondents. Next we turn to validation of the index for perceptions of the usefulness of a set of possible "Chunking" tools. Frequency distributions for the variables in the study are presented, followed by the use of correlations and regression analysis to test the hypotheses. We complete this section with a discussion of the results.

\subsection{Characteristics of Respondents and their Agencies}

Most of the respondents work for agencies with few staff members ( $85.5 \%$ have only 1 to 9 employees) that serve small population counties $(61 \%$ have less than 50,000 residents). Note that although in general, the large counties have more staff, that relationship is not ubiquitous. A little over half of the respondents are over 50 years of age and $60 \%$ have worked for five years or more at their current agency. Most of the respondents who answered the question regarding their personal and work use of SM $(\mathrm{N}=90)$ have had some experience using SM (98.7\%). Only six respondents reported having never used SM. On a scale from 1 (never) to 7 (frequently), the reported use of SM had a 
mean of 5.28 and a median of 6. Thus, our respondents were at least somewhat familiar with SM.

\subsection{Factor Analysis and Reliability Test for Chunking Software Tools}

The factor analysis results were that two factors were extracted with six items in first factor and four items in the second factor. All five items related to categorizing social media load on the first factor, which we have labeled "Chunking". All loadings for the two factors exceed 0.6 , with a particularly high threshold of 0.73 for first factor, and there was no cross construct loading that exceeded 0.6. In order to keep the Chunking factor "clean" in addressing the issue of usefulness of categorizing SM, we dropped the "integrate" item, ("During a crisis how useful would it be to have a way to automatically integrate social media data (e.g. Twitter feeds shown on a map) into any major information system you currently use for information?") because it is not conceptually part of "chunking" or "categorization". We then assessed convergent validity by using Cronbach's alpha. Reliability analysis was used to assess the consistency of the factor. According to Hair et al. [5], the widely accepted lower limit for Cronbach's alpha is .70. As shown below in Table 1, Cronbach's alpha for the factor has a high coefficient of .923 .

Table 1. Factor analysis result for "Chunking"

\begin{tabular}{|c|c|c|c|}
\hline \multirow[t]{6}{*}{$\begin{array}{l}\text { 1st } \\
\text { factor }\end{array}$} & Items & $\begin{array}{l}\text { Factor } \\
\text { loadings }\end{array}$ & $\begin{array}{l}\text { Cronbach's } \\
\text { Alpha }\end{array}$ \\
\hline & $\begin{array}{l}\text { Filter: There are now systems that have been developed in research projects that } \\
\text { can use a combination of Natural Language Processing and expert ratings to filter } \\
\text { incoming social media posts for relevancy to a disaster, identify the major } \\
\text { categories represented in the data and classify them in that way. How useful do } \\
\text { you think such as system would be viewed by your agency? }\end{array}$ & .784 & \multirow[t]{5}{*}{.923} \\
\hline & $\begin{array}{l}\text { Category: During a crisis would being able to view social media data as } \\
\text { generated by categories of users like NGOs only, local government } \\
\text { representatives only, local residents/ beneficiaries in the affected areas only, etc. } \\
\text { be seen as useful to your organization? }\end{array}$ & .779 & \\
\hline & $\begin{array}{l}\text { Map: Would being able to view social media data as generated by geographical } \\
\text { location and some form of map- based display of social media data during a crisis } \\
\text { be seen as useful to your organization? }\end{array}$ & .852 & \\
\hline & $\begin{array}{l}\text { Subevent: During a crisis, would being able to view social media data as } \\
\text { categorized by subevents such as rescue issues, recovery issues, mobilization } \\
\text { issues, infrastructure issues, etc. be seen as useful to your organization? }\end{array}$ & .801 & \\
\hline & $\begin{array}{l}\text { Folder: How useful would it be to your agency to be able to create a customizable } \\
\text { "folder" for your agency in which a set of social media profiles or threads (e.g. } \\
\text { Twitter subscriptions) was made to meet your agency's needs for each disaster } \\
\text { response? }\end{array}$ & .734 & \\
\hline
\end{tabular}

Table 2. Frequency distribution of variables

\begin{tabular}{|l|c|c|c|c|c|c|c|c|c|c|}
\hline Items & Mean & S.D. & 1 & 2 & 3 & 4 & 5 & 6 & 7 & $\#$ \\
\hline BIO & 3.83 & 2.14 & $23.5 \%$ & $9.1 \%$ & $12.4 \%$ & $13.7 \%$ & $13.3 \%$ & $13.3 \%$ & $14.8 \%$ & 460 \\
\hline Filter & 5.53 & 1.58 & $2.2 \%$ & $4.6 \%$ & $4.6 \%$ & $11 \%$ & $16.6 \%$ & $25.4 \%$ & $35.5 \%$ & 409 \\
\hline Category & 5.71 & 1.42 & $1.4 \%$ & $1.9 \%$ & $3.5 \%$ & $13.0 \%$ & $17.1 \%$ & $25.7 \%$ & $37.5 \%$ & 432 \\
\hline Map & 5.99 & 1.29 & $1.4 \%$ & $.9 \%$ & $2.5 \%$ & $7.3 \%$ & $16.6 \%$ & $26.4 \%$ & $44.9 \%$ & 439 \\
\hline Subevent & 5.77 & 1.41 & $1.8 \%$ & $1.4 \%$ & $3.2 \%$ & $11.6 \%$ & $18.3 \%$ & $24.7 \%$ & $39 \%$ & 438 \\
\hline Folder & 5.67 & 1.44 & $2 \%$ & $2.3 \%$ & $2.8 \%$ & $11 \%$ & $19.7 \%$ & $25.6 \%$ & $36.6 \%$ & 391 \\
\hline IU & 5.50 & 1.63 & $2.2 \%$ & $2.2 \%$ & $6.7 \%$ & $20 \%$ & $13.3 \%$ & $12.2 \%$ & $43.4 \%$ & 90 \\
\hline
\end{tabular}

Note: S.D.=standard deviation; 1 to 7 are seven numeric from semantic differential scale; \# = Number of Responses

\subsection{Frequency Distributions for All Variables in the Study}

Table 2 shows the overall distributions of the variables of interest in this paper. Perceptions of the importance of Information Overload are dispersed: almost a quarter do not see it as a barrier at all, but about $40 \%$ do perceive it as an important barrier (rating $\mathrm{BIO}$ at 5,6 , or 7). The five types of Chunking tools are generally perceived as useful; we then aggregated them 
by taking the mathematical average of the five items to compute the new "Chunking" variable (Mean=5.73, $\mathrm{SD}=1.43$ ), for further analysis .

Ratings of the usefulness of the Chunking tools are highly skewed in the positive direction. All five of the tools are rated at 6 or 7 on the 7-point scale, by a majority of the respondents. While one might expect that information overload would be seen by younger respondents as less of a barrier than is perceived by older respondents, a Chi-Square test indicates that perceptions of BIO do not vary significantly by age $(\mathrm{p}=.297)$.

The respondents highly rated their intention to use SM in their work, if possible, $(\mu=5.5$ on the 1 to 7 scale, $\mathrm{N}=90$ ). Although many of the survey respondents did not answer this question, perhaps because it was placed at the end of the survey, the responses from those who did answer indicate an awareness of the benefits of SM and a hope to be able to use it in their agency.

\subsection{Correlation and Regression Results}

The correlations of the variables studied to test our hypothesis are listed in table 3 .

Table 3. Correlations among variables

\begin{tabular}{|l|l|l|l|}
\hline Correlations & $\mathrm{BIO}$ & Chunking & IU \\
\hline BIO & 1 & & \\
\hline Chunking & -.02 & 1 & \\
\hline IU & $-.26^{*}$ & $.61^{*}$ & 1 \\
\hline Note. ${ }^{*} p<.05$. & \\
\hline
\end{tabular}

The Perceived Barrier of Information Overload is significantly and negatively correlated with Employees' intention to use of social media (Pearson's $r=-.26)$. Perceived Usefulness of Chunking tools is positively related to Intention to Use social media (Pearson's $r=.58$ ).

In this research, multiple regression was used as an extension to simple correlation between variables, aiming at addressing the issues of causality and interaction. As Table 4 shows, the first test shows that BIO alone explains $5.4 \%\left(\mathrm{R}^{2}=.054, \beta=-.256 \mathrm{p}=\right.$ $.017)$ of the variance of the dependent variable. The second test shows that the perceived usefulness of Chunking considered alone explains 35.8 percent $\left(\mathrm{R}^{2}=\right.$ $.358, \beta=.606, p=.000)$ of the variance of the dependent variable. The third test, which was a multiple regression of the two independent variables on the dependent variable of Intention to Use, explains 38 percent $\left(\mathrm{R}^{2}=.380\right)$ of the variance of the dependent variable, which is higher than either independent variable considered alone.
Table 4. Results of three regression tests. Dependent variable is intention to use (IU).

\begin{tabular}{|l|l|l|l|l|}
\hline $\begin{array}{l}\text { Independent } \\
\text { variables }\end{array}$ & $\mathrm{R}^{2}$ & $\mathrm{~F}$-value & $\begin{array}{l}\text { Standard } \\
\text { coefficient( }(\beta)\end{array}$ & Sig. \\
\hline BIO & .054 & 5.877 & -.256 & .017 \\
\hline Chunking & .358 & 42.913 & .606 & .000 \\
\hline BIO & .380 & 23.390 & -.205 & .000 \\
Chunking & & & .581 & .029 \\
.000
\end{tabular}

Thus, $\mathrm{H} 1$ and $\mathrm{H} 2$ are supported. However, a regression test found that BIO is not a significant predictor of perceived usefulness of Chunking $(\mathrm{p}=.686)$. Hypothesis 3 is not supported.

\subsection{Discussion}

The extent to which information overload is perceived by U.S. county-level emergency managers as a barrier to the use of social media to collect information during an emergency (BIO) varies considerably; almost a quarter do not see it as a barrier at all, but about $40 \%$ do perceive it as an important barrier. The extent to which information overload is considered to be a barrier predicts, negatively, the intention to use SM on the job. (H1 is supported). That is, the more a respondent perceives information overload as a barrier to use, the less likely it is that they intend to use SM on the job. On the other hand, the extent to which the five "Chunking" tools to deal with incoming social media posts are considered to be useful positively predicts the intention to use social media on the job (H2 is supported). That is, the more a respondent perceives Chunking tools as useful, the higher their intention to use SM on the job. In addition, regression analysis shows that using both of these variables simultaneously improves the prediction of Intention to Use of SM.

The managers are enthusiastic about the potential usefulness of Chunking types of software tools to manage incoming information from social media. A high priority should be placed by software developers working in this field on agreeing on a standard "toolkit," moving the systems from research or prototype systems to production systems ready for realtime use in disaster management, and integrating these tools into the systems now used by emergency managers.

However, the level of BIO is not shown to predict significantly the level of perceived usefulness of Chunking technologies (H3 is not supported). This may be a result of the respondents' overwhelmingly 
positive appreciation of the usefulness of chunking technologies.

\section{Limitations and Contributions}

\subsection{Limitations}

As with all research studies, there are limitations to be noted with this study. Only one method (survey) was used in this study. Keeping the survey short also resulted in our inability to address related but important issues such as policy and respondent's ideas about enhancements that they would like to see. In addition, we only surveyed U.S. county-level emergency managers, which limits the generalizability of this study. Unfortunately, although many countylevel emergency managers did take the survey, a good proportion of them did not answer all questions. Fortunately, all questions did have an adequate (over 30) number of responses so analysis could be performed.

\subsection{Contributions}

These data empirically confirm previous statements that Information Overload is a problem that presents a barrier to use of social media during management of emergencies. However, it also points the way forward to solutions to this problem. This research contributes to extant literature by creating the beginning of a bridge between developers of technology to enhance SM data use and the potential users of those technologies in crisis situations. That is, it examines the perceptions of potential users of these enhancements (county- level EMs in the U.S.) about their usefulness, and shows that these perceptions are positively related to intention to use social media on the job in the future. This research also adds clarity to the issue of information overload as a barrier to making use of the wealth of social media data during crisis. This is an important contribution as, if that data could be used effectively, situational awareness can be improved for emergency managers.

Additionally, this is the first research, to our knowledge, that shows that Miller's 1956 "Magical Number" work [16] is applicable to the study of improving SM usefulness for emergency management and ameliorating the issue of information overload. This can provide guidance to both technologists and researchers as they work to find ways to improve the presentation of SM data so that the wealth of information on it can be effectively and efficiently used.

\section{Future Research}

It is important to do further analysis to assess whether some variables representing characteristics of the agency and/or respondent have either confounding or moderating effects on the relationships reported in this paper. In addition, it would be useful to have data on government emergency managers at different levels (e.g., state and federal) as well as at the local county level, on emergency managers who work through NonGovernmental Organizations, and on emergency managers of all types from nations other than the U.S. Finally, in this paper we have examined only the barrier of perceived information overload; in future analyses we plan to also examine other important barriers and how they may be ameliorated.

Additionally, we are currently in the planning and fund-seeking stage of an effort to bring together technologists and EM managers who could benefit from SM technology enhancement efforts. This project would enable the technologists to better understand the actual needs and desires of the EM SM users and enable the EM users to understand better what is technologically feasible. The project wwould include workshops (with prototype demonstrations if possible) and other collaborative activities such as web discussions and Delphi exercises.

\section{Acknowledgements}

We wish to thank our colleagues Dr. Jane Kushma and Dr. Andrea Tapia for their work on the research instruments. We especially thank the EM professionals who took time from their very busy schedules to complete our survey.

\section{References}

[1] Cameron, M.A., Power, R., Robinson, B, and Yin, J. (2012). Emergency situation awareness from Twitter for crisis Management. Proceedings of the WWW 2012- SWDM ' 12 Workshop, April 16-20 2012, Lyon, France, ACM., 695698.

[2] Collins, R., Dwyer, C., Hiltz, S., \& Shrivastav, H. (2012). Do I know what you can see? Social networking sites and privacy management. In Proceedings of AMCIS 2012.

[3] Eppler, M.J. \& Mengis, J. (2003) A Framework for Information Overload Research in Organizations. Università della Svizzera italiana, paper \#1/2003.

[4] Eppler, M.J. \& Mengis, J. (2004) The Concept of Information Overload: A Review of Literature from 
Organization Science, Accounting, Marketing, MIS, and Related Disciplines. The Information Society, 20, 325-344.

[5] Hair, J.F., Black, W.C., Babin, B.J., Anderson, R.E., \& Tatham, R.L.(2006). Multivariate data analysis, 6th Ed. Upper Saddle River, NJ: Pearson Prentice Hall

[6] Hiltz, S.R. and Plotnick, L. (2013) Dealing with information overload when using social media for emergency management: Emerging solutions. In T. Comes, F. Fiedrich, S. Fortier, J. Geldermann \& T. Müller (Eds.), Proceedings of the 10th International ISCRAM Conference, Baden Baden, Germany.

[7] Hiltz, S.R. and Turoff, M. (1985) Structuring ComputerMediated Communication to Avoid Information Overload, Communications of the ACM, 28, 7, 680- 689.

[8] Imran, M, Castillo, C., Diaz, F. and Vieweg, S. (2015). Processing Social Media Messages in Mass Emergency: A Survey. ACM Computing Surveys, 47, 4 Article 67.

[9] Imran, M., Castillo, C., Elbassuoni, S., Diaz, F. \& Meier, P. (2013). Extracting information nuggests from disasterrelated messages in social media In T. Comes, F. Fiedrich, S. Fortier, J. Geldermann \& T. Müller (Eds.), Proceedings of the 10th International ISCRAM Conference (pp. 791-801). Baden Baden, Germany.

[10] Imran, M., Castillo, C., Lucas, J., Meier, P., \& Rogstadius, J. (2014). Coordinating human and machine intelligence to classify microblog communications in crises. In Proceedings of the 11th International ISCRAM Conference (pp. 712-721). University Park, PA.

[11] Imran, M., Elbassuomi, S., Castillo, C., Diaz, F. and Meier, P. (2013b). Practical extraction of disaster-relevant information from social media. WWW 2013 Companion, May 13-17, 2013, Rio de Janeiro, Brazil.

[12] Koroleva, K., Krasnova, H., \& Günther, O. (2010) 'STOP SPAMMING ME!' - Exploring Information Overload on Facebook. In Proceedings of AMCIS, August 12- 15; Lima, Peru.

[13] Landau, S., and Everitt, B. S., A Handbook of Statistical Analyses Using SPSS, Chapman \& Hall/CRC; 2004.

[14] Liu, S.B.; Palen, L.; Sutton, J.; Hughes, A.L.; \& Vieweg, S. (2008). In search of the bigger picture: the emergent role of on-line photo sharing in times of disaster. In Proceedings of the Fifth International ISCRAM Conference, 140-149. Washington, DC, May 4-7. Available at www.iscram.org.

[15] MacEachren, A.M., Jaiswall, A., Robinson, A.C., Pezanowski, S., Savelyev, A., Mitral, P., Zhang, X., \&
Blanford, J. (2011). SensePlace2: GeoTwitter analytics support for situational awareness. In Proceedings of IEEE Symposium on Visual Analytics Science and Technology, Providence, RI.

[16] Miller, George,1956. The Magical Number Seven, Plus or Minus Two: Some Limits on Our Capacity for Processing Information. Psychological Review 63 (2): 81-97.

[17] Plotnick, L., Hiltz, S.R., Kushma, J. \& Tapia, A. (2015). Red tape: Attitudes and issues related to use of social media by U.S. county-level emergency managers. In Proceedings of ISCRAM 2015, Kristiansand, Norway

[18] Sheridan, T.B. \& Ferrell, W.R. (1974). Man-Machine System: Information, control, and decision models of humans performance. Cambridge, Mass.: MIT Press.

[19] Starbird, K. (2011). Digital volunteerism during disaster; Crowdsourcing information processing. In Proceedings of '11 Workshop on Crowdsourcing and Human Computation, Vancouver, BC. the 2011 Conference on Human Factors in Computing Systems (CHI 2011) (pp. 1071-1080). Vancouver, BC: ACM.

[20] Tegarden, D.P. (1999) Business Information Visualization. Communications of the Association for Information Systems, Volume 1, Paper 4.

[21] Verma, S., Vieweg, S., Corvey, W.J., Palen, L., Martin, J.H., Palmer, M., Schram, A., and Anderson, K.M. (2011) Natural Language Processing to the Rescue: Extracting 'Situational Awareness' Tweets during mass emergency, Proceedings of the Fifth International AAAI Conference on Weblogs and Social Media, 385- 392.

[22] Yin, J., Karimi, S., Robinson, B., \& Cameron, M. (2012b). ESA : Emergency situation awareness via microbloggers. In Proceedings of CIKM '12 (pp. 27012703).

[23] Yin, J. Lampert, A., Cameron, M., Robinson, B. \& Power, R. (2012a). Using social media to enhance emergency situation awareness. IEEE Intelligent Systems, Nov/Dec. 5259.

[24] St. Denis, L.A., Palen, L. \& Anderson, K.M. (2014) Mastering Social Media: An Analysis of Jefferson County's Communications During the 2013 Colorado Floods, Proceedings of the $11^{\text {th }}$ International ISCRAM Conference (pp. 737-746).

[25] Hiltz, S.R., Kushma, J. \& Plotnick, L. (2014) Use of Social Media by U.S. Public Sector Emergency Managers: Barriers and Wish Lists, Proceedings of the $11^{\text {th }}$ International ISCRAM Conference (pp. 602-611). 